\title{
Desktop Scanner Metrology
}

\author{
Pavel Divis, Gordon M. Mair, and Jonathan Corney \\ University of Strathclyde, Glasgow, UK
}

\begin{abstract}
An investigation is presented of a low cost approach to the measurement of two and three dimensional objects using a flatbed scanner and image analysis software. Conventional measurement using relatively low cost instruments such as micrometers and vernier callipers can be time consuming and requires operator skills which result in higher overall costs. The increasing resolution and decreasing prices of flatbed scanners introduces the possibility of their use as a low cost alternative to traditional manual measuring. To investigate this, a simple dimensional measurement technique was developed using an unmodified, then a modified, flatbed scanner, a standard PC, and software. A dimensional accuracy of $\pm 0.05 \mathrm{~mm}$ was achieved with a modified flatbed scanner system for slip gauge samples of nominal thickness $10 \mathrm{~mm}$ and $5 \mathrm{~mm}$.
\end{abstract}

\section{Introduction}

It has been estimated that $3 \%$ to $6 \%$ of the GDP of industrialised countries is spent on measurement and measurement related operations [1]. Even minor contributions towards reducing this cost will be worthwhile. This paper therefore presents an investigation into the possibility of utilizing a commercially available flatbed scanner for basic component metrology. For the measurement of simple components smaller than $100 \mathrm{~mm}$ the use of conventional low cost methods using manual instruments such as vernier callipers (precision $+/-0.01 \mathrm{~mm}$ ) or, micrometers (precision $+/-0.005 \mathrm{~mm}$ ) is time consuming and requires operator skills which result in high total costs. For the accurate measurement of more complex components, digital coordinate measuring machines (CMMs) are more commonly used (precision $+/-0.0005 \mathrm{~mm}$ ) but these are relatively expensive. In order to investigate the possibility of a low cost, low skill, mechatronic metrology system, the work reported here focuses on finding the accuracy that can be obtained from a flatbed scanner.

\subsection{Previous Applications of Flatbed Scanners}

The flatbed scanner (FBS) has been used in various scientific applications during the last ten years due to its increasing capability to capture images in high resolution and the substantial reduction of computation costs. An FBS has been used for automatic examination of damaged organs in medicine [2,3] and they have been used for automated evaluation of organic forms such as detection of leaf areas and identification of conifer needle geometry [4,5], monitoring of root growth [6], and for quantification of microbial growth [7]. Although these studies focused on percentage comparison of different colour ranges or measurement of area by counting high/low intensity pixels 
they did not provide a full investigation of the FBS accuracy for dimensional measurement. Several material and civil engineering applications utilising an FBS have also been developed for determination of spray droplet size [8], analysis of air voids in concrete [9], and archaeological mortar porosity [10]. These applications acquired a planar image within the focus field of the scanner. Other FBS applications are to be found in the food industry where scanned images were used for automatic inspection of broken rice kernels [11] size and colour grading of lentils [12] and food texture grading [13]. Elsewhere 100x100 mm plates of air entrained concrete were scanned [14] and the longitudinal and lateral distortion of a scanned image across large dimensions was examined [15]. Two particularly relevant and recent FBS applications are notable; in one paper section lengths of injection moulded test spirals were measured [16], and in another automatic wire diameter measurements were made using a Matlab image processing toolkit [17]. The main disadvantage of these systems is that they were developed for measurements that were almost 2D. The edge detection methods used would be extremely difficult to employ for accurate edge finding of 3D objects since when the FBS captures an image of a non-planar object the side surface of the object may be captured together with the front face, see Figure 1.

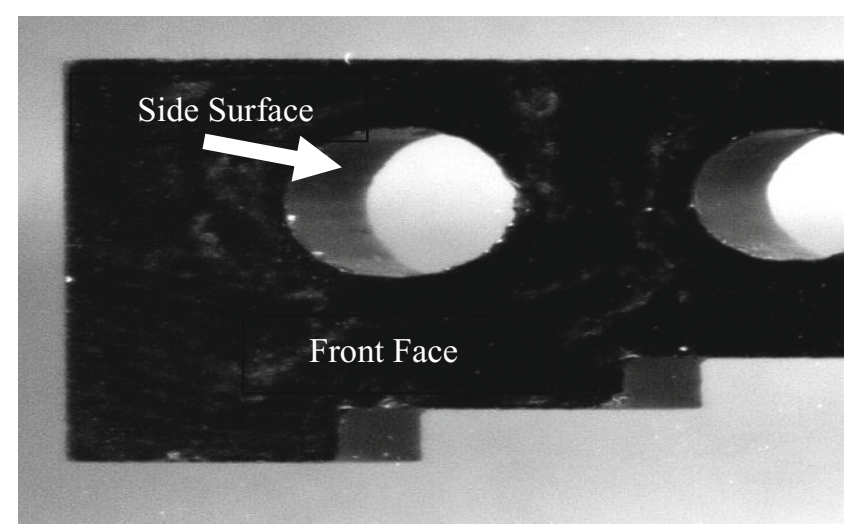

Fig. 1. FBS captured both front view and side view image

It is difficult for an automatea _. cognition system to distinguish which edge belongs to the front face and which belongs to the side face therefore the dimensional measurements would be highly dependent on orientation and position of the object.

\section{Initial Experiment and Methodology}

\subsection{Introduction}

In the initial experiment no hardware modifications were made. A simple planar component was chosen to be scanned in order to determine how scanner resolution, component orientation, component distance from the scanner surface and component size 
influenced accuracy. There are several experiment designs that could have been used for evaluation of the results such as full factorial design, randomized block design, Latin square design or Taguchi experimental design methods. The Taguchi method was selected for the following reasons. (i) Fractional factorial experiments using Taguchi statistical methods of orthogonal arrays require relatively few runs and can still provide a reliable outcome. (ii) At the end of the data evaluation the outcome of Taguchi's method would be a list of factor levels that will provide the best possible performance of the flatbed scanner. (iii) The experiment information can also be used for determination of the FBS limits in terms of precision and resolution. The advantages of the fractional factorial designs at two levels have also been noted [18]. In this paper, due to space restrictions, only examples and summary results are included however they should provide a good indication as to the procedures followed and the results obtained.

\subsection{Image Processing Software}

Image processing utilised ImageJ [19] open architecture image analysis software that can be linked with MS Excel. Custom acquisition, analysis and processing plug-ins were developed using a built-in editor and Java compiler. This software was used for three reasons; good image processing which was simple for modification; programmable calculation capabilities including a wide range of inbuilt algorithms; and freeware availability. With regard to analysing the image the most suitable software function is edge detection. The image edge may be defined as "the boundary between two regions separated by two relatively distinct grey level properties" [20]. Although the accuracy with which the computer is able to recognise an edge is a crucial factor in system performance [21], there is no index that would help to decide what method should be used for a particular application and the performance of an edge detection method is always evaluated subjectively according to user needs [22].

The edge detection used in this experiment followed Goodman [23] who mathematically and experimentally proved that for two dimensional objects in coherent light the geometrical position of the edge refers to the image coordinate where the light intensity is $25 \%$ of its value in the illuminated area far from the edge. Therefore if the intensities of all pixels are summed in a vertical direction, the average edge position can be identified by drawing a threshold line at $75 \%$ dark pixels. To accurately determine the boundary of the measured component from the FBS scanned image, the binary image can be prepared by defining a range of brightness values in the original grey scale belonging to the component body and rejecting all of the other pixels to the background. This is the thresholding operation [24] and it is relatively straight forward. ImageJ $1.42 q$ software, developed by the USA National Institute of Health, was used to sum up the vertical pixel intensities. Data from this software were exported to MS Excel where the data analysis algorithm was programmed in a Visual Basic (VB) environment.

\subsection{Variables Considered}

Resolution (R). High resolution flatbed scanners can currently be purchased with hardware resolution of 6400dpi and above. Theoretically this would result in a 
measurement accuracy better than $0.004 \mathrm{~mm}(4 \mu \mathrm{m})$ however the scanning speeds of such scanners are too slow for our purposes. The Epson Perfection 350 scanner [25] used for the initial experiment had a resolution of 4800dpi hence two levels of this resolution factor were chosen to compare significance of the flat bed scanner resolution factor on measurement accuracy. These were 1200dpi resolution shown as (-), and 4800dpi resolution shown as (+) in Table 1.

Table 1. Initial experiment matrix including results

\begin{tabular}{|c|c|c|c|c|c|c|}
\hline 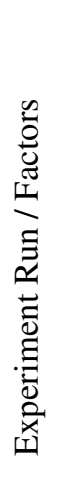 & 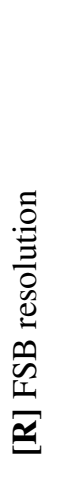 & 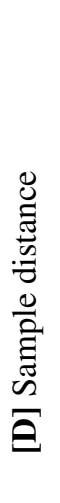 & 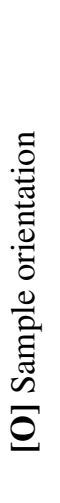 & 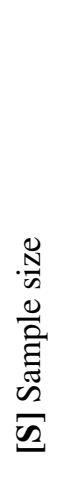 & 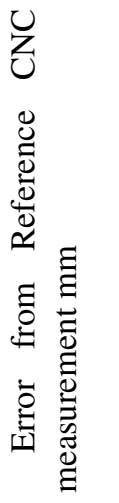 & 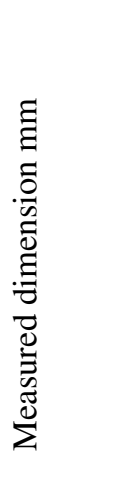 \\
\hline 1 & - & - & - & - & 0.712 & 0.296 \\
\hline 2 & + & - & - & + & 0.037 & 13.457 \\
\hline 3 & - & + & - & + & 0.497 & 12.996 \\
\hline 4 & + & + & - & - & 0.246 & 0.762 \\
\hline 5 & - & - & + & + & -0.053 & 13.547 \\
\hline 6 & + & - & + & - & 0.320 & 0.688 \\
\hline 7 & - & + & + & - & -0.029 & 1.037 \\
\hline 8 & + & + & + & + & 0.529 & 12.965 \\
\hline
\end{tabular}

Distance of Test Piece from FBS (D). The majority of the products measured in manufacturing metrology are three-dimensional and the product edges are not in the same plane, therefore the "distance of the object" factor investigates the FBS sensitivity to the object location. Results analysis was carried out for the component resting directly on the glass platen and at a distance of $10 \mathrm{~mm}$ from the surface, in Table 1 these are shown as (-) and (+) respectively. Light dispersion due to placement of the object out of the focal plane is a well-known topic [26]. The edges of a scanned object will be increasingly blurred depending on the distance from the focal plane. The FBS scans an image line by line (of pixels) the actual intensity of the light falling on one particular sensor cell is obtained from the average light intensities above the cell. All scanner manufacturers adjust the optical system of the FBS so that it produces sharp images if the sheet of paper is placed on top of the FBS glass platen.

Test Piece Orientation (O). Another significant factor to consider for the accuracy evaluation of the FBS is the angle at which the component is scanned. Possible image 
processing errors that may occur during the image recalculation are described in the Matlab Image Processing Toolkit handbook [27]. Moreover as flatbed scanners often have different longitudinal and lateral resolutions, it is appropriate to assume that this factor can be a source of measurement error. Therefore, two separate levels of this factor were examined in order to find out its significance. One with the slip gauge placed parallel with the scanning head movement, (-) in Table 1 and another with the slip gauge physically tilted by 30 degrees, shown as level (+). The latter image was automatically rotated to the parallel position via ImageJ software. This process of software-based rotation might be a source of error since all pixels in the image matrix need to be recalculated with respect to the perpendicular orientation.

Object Size (S). The final possibly significant factor that may introduce error to the scanner measurement is the size of the object. In the case of smaller samples the pixels are likely to be equally distributed across the image whereas if scanning larger objects the pixel density might not be equal especially in the lateral direction.

The longitudinal distortion is predominantly caused by the scanning head carriage displacement, either due to an uneven speed or to a misaligned movement. The lateral distortion is caused by imperfections on the internal cylindrical lenses of the scanner, although the scanner manufacturer usually calibrates these by using a sequence of images with different chromatic filters. The effect of the sample size on the measurement accuracy should be minimal and will be more likely affected by the light source or sample orientation. Two sizes were used on the thin sheet steel stencil a strip of $13.5 \mathrm{~mm}$ and a slot of $1 \mathrm{~mm}$, shown as ' $a$ ' and ' $b$ ' in Figure 2 and as factors (+) and (-) respectively in Table 1 .

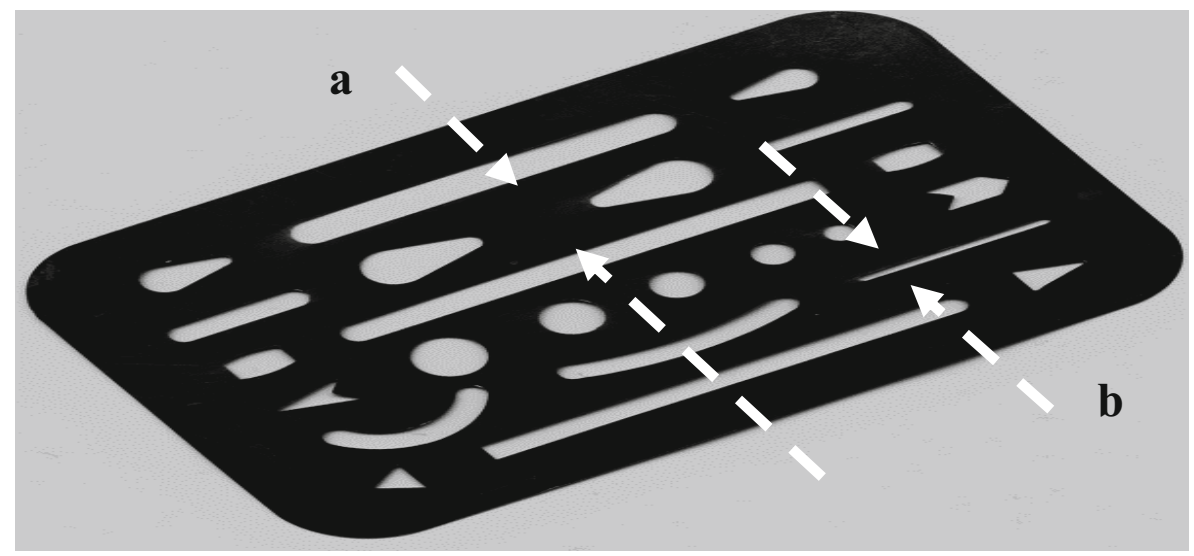

Fig. 2. Measured dimensions $a=13.5 \mathrm{~mm}$ and $b=1 \mathrm{~mm}$

A datum value for the two dimensions was determined by the 3D CNC Optical Measuring Machine, a Mitutoyo 'Quick Scope'. This is a calibrated machine with a resolution of $0.5 \mu \mathrm{m}$ and an accuracy of $2.5+6 \mathrm{~L} / 1000 \mu \mathrm{m}$. The slot width was calculated from the average value of 200 automatically generated widths in a length of $2 \mathrm{~mm}$. There were six values acquired across the slot length. The three slot widths, at 
left, middle and right side of the slot and its parallelism at these three locations. An average of the three measurements was used as a benchmark value to the readings obtained with the FBS.

\subsection{Initial Experiment Results}

The average intensities for each pixel column using the relatively low resolution images (1200dpi) are shown in Figure 3. A plot was also obtained for the higher resolution scans. Because the scanned object very often projected a shadow onto the white board behind the component causing sudden peaks in the graphs, the brightness values were processed through the rolling average of 15 pixels light intensity values to smooth the curves.

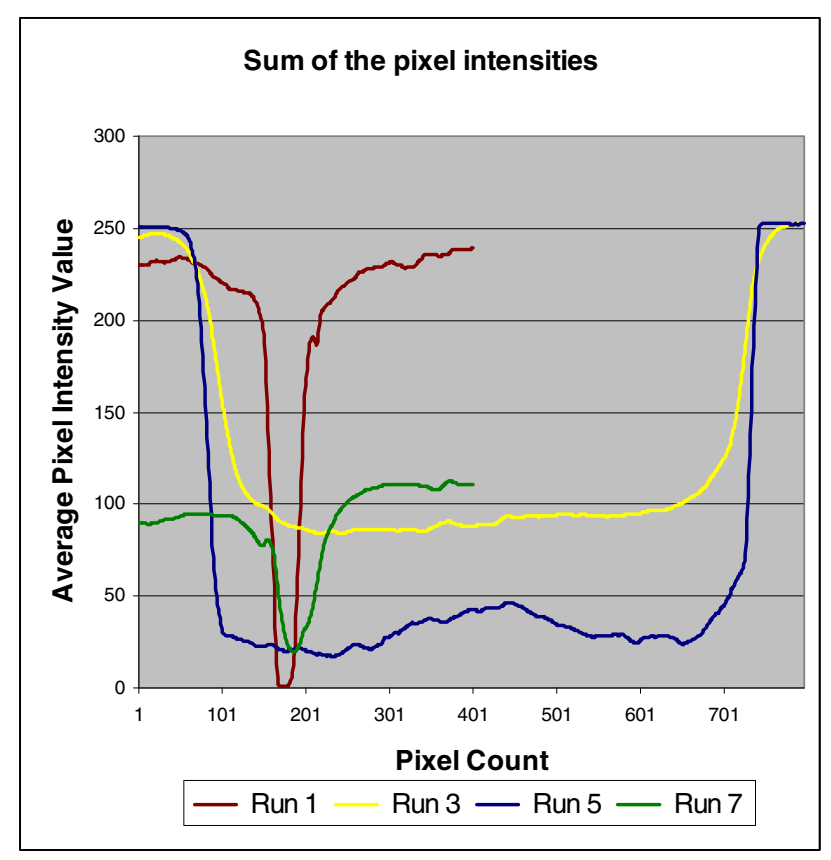

Fig. 3. Average pixel intensities for a column of the pixels, low resolution scans

In this experiment the key measurement quality was dimensional accuracy. Dimension results obtained from the image analysis algorithm, were compared with the dimensions acquired by reference measurements from the $\mathrm{CNC}$ vision system. The differences between FBS and $\mathrm{CNC}$ readings were taken as inputs for the statistical analysis.

\subsection{Initial Experiment Comments}

The significance of the factors of resolution, distance, orientation, and size, and their interactions were calculated and it was found that the flatbed scanner without any modification is able to measure within accuracy of $\pm 0.19 \mathrm{~mm}$ which is one standard 
deviation of all measurement results. The most significant factor interaction that negatively influenced the accuracy was the distance of the scanned object interacting with the object size. For instance, in the case an object of small dimensions scanned at a distance of $10 \mathrm{~mm}$ from the platen a poor measurement accuracy of $\pm 0.39 \mathrm{~mm}$ could be expected. Such a large effect can be explained by the low focal field of the FBS which means that if a narrow component is placed outside the field its edges appear very blurred and accurate detection of the object edge becomes more difficult. Accordingly placing wider object outside the FBS focal plane generates clearer images where component edges are easier to be defined by automatic image processing. The best measurement accuracy of the $13.5 \mathrm{~mm}$ width object placed directly on the FBS glass was $0.06 \mathrm{~mm}$ which is very close to the scanner hardware limits at $1200 \mathrm{dpi}$.

\section{Second Stage Experiment}

An accuracy of $\pm 0.19 \mathrm{~mm}$ for the initial experiment using an unmodified FBS is too low for metrology applications and therefore some modifications to the FBS were introduced to diminish image errors caused by the following factors identified during the initial experiment:

- $\quad$ Blurred edges of the components placed out of the FBS focus field.

- $\quad$ Viewing angle of the CCD matrix.

- $\quad$ Light dispersal from the component sides.

- $\quad$ Shadow projected behind the component by the scanner lamp.

For the second stage of the experiment an Epson Perfection 1260 FBS was used. This scanner had a hardware resolution of $1200 x 1200 d p i$. In this experiment two levels of scanner resolution [R] were again used but this time they were 600dpi and 1200dpi.

\subsection{Lighting of the Component [L]}

Initially a Super Flux PCB white LED [28] was used as spot light source. The maximum luminosity of the LED was tested in the Department laboratory and was achieved at $4.8 \mathrm{~V}$ and $100 \mathrm{~mA}$ which made it ideal for power source from PC USB. This was held at a distance of $100 \mathrm{~mm}$ from the scanning surface by a multipurpose laboratory holder, see Figure 4.

One of the key causes of the measurement error in the first experiment was shadows behind the component caused by the scanner lamp. The reflections from the component created white areas that misled the image analysis algorithm.. Therfore for the alternative light source the cold cathode fluorescent lamp was taken from the scanning head and mounted on a gantry above the platen, again at a height of $100 \mathrm{~mm}$. This illuminated the component from above and projected the shadow of the component onto a diffuser layer placed on the FBS platen thus allowing the shadow to be scanned by the FBS, see Figure 5 . 


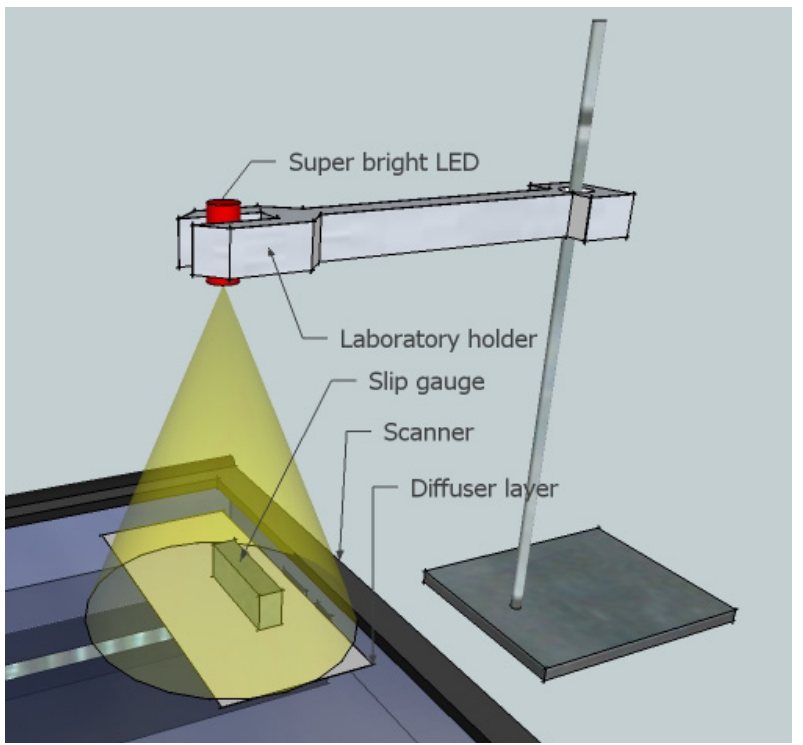

Fig. 4. Light source at level (-) causing inaccuracy

The first the light source at level (-) is the LED spot light and at level (+) we have the parallel beam created by the Cold Cathode Fluorescent Lamp (CCFL) removed from the scanner chassis. The CCFL generates light that spreads in a cylindrical direction and therefore all light beams perpendicular to the lamp axis are emitted in parallel planes. Therefore it is appropriate to assume that the component dimensions parallel with the lamp are illuminated with parallel rays of light.

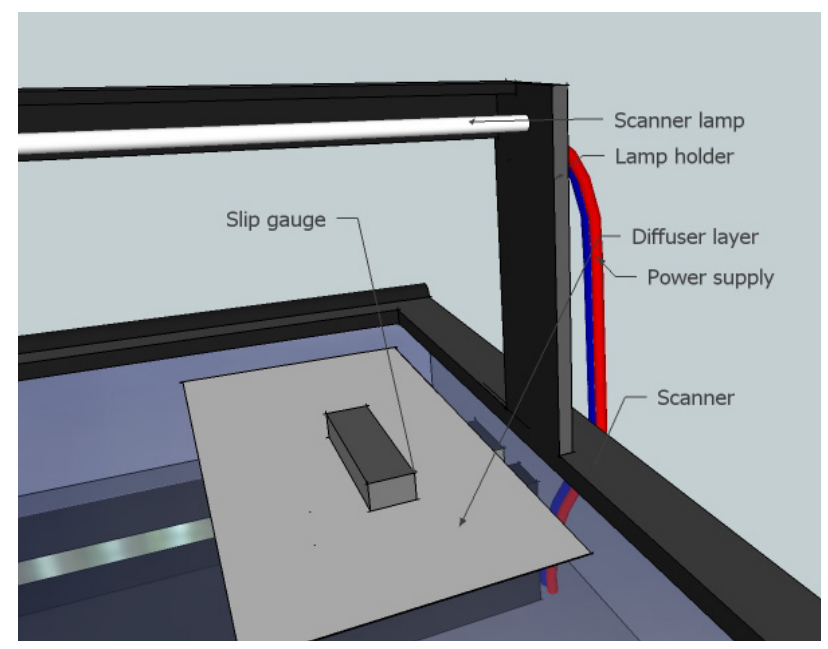

Fig. 5. Light source at level (+), cold cathode fluorescent lamp light source placed above the slip gauge creating a parallel source of light 
The addition of a diffuser layer on the FBS platen has been already proven to be significant see [15] where it is stated that "the denser and thinner the plate of the diffuser and the smaller the particle, the finer the image measured". Also care has to be taken attaching the diffusion plate onto the surface of the scanner image screen to avoid wrinkles or tilts as it may result in distortion of the scanned image. There are two possibilities of the diffuser layer application; it can be in the form of thin film, or diffusion paint can be used. However with the second option it is difficult to maintain an equal thickness of the paint across the scanned area. In this project a diffusion layer of translucent polyethylene of $0.05 \mathrm{~mm}$ thick was used. This polymer has an amorphous structure which would ensure equal distribution of the shadow and extremely fine grain of the image.

\subsection{Other Factors}

The effect of the light deflection at the edge of an illuminated component grows with the component distance from the diffuser layer. Effects of the light deflection at the edge of any solid object have been described elsewhere, see [29] where the projection of 3D objects onto a diffuser layer is addressed. As noted earlier it is known that in the case of two dimensional objects the geometrical position of the edge refers to the image coordinate where the light intensity is $25 \%$ of its value in the illuminated area far from the edge. However a different situation takes place for three dimensional objects during the shadow projection. A relatively simple approach developed by Chugui [30] for edge position determination of extended bodies reduces the problem into an analysis of a planar profile object located in space. It is expected that when the distance of an object from the diffuser layer is increasing the projected shadow loses its sharpness. Therefore in order to investigate the relationship between the component position in space and the accuracy of the measured size, the "distance of the object" [D] factor is investigated in the experiment. Two different levels of this factor are used in the experiment. The level (-) of this factor refers to the slip gauge placed directly onto the diffuser layer, illustrated and level (+) of this parameter refers to the slip gauge at a distance of $10 \mathrm{~mm}$ from the diffuser layer.

Other factors assessed were: sample orientation [O] where (-) indicates sample parallel to the scanning head and [+] at $30^{\circ}$ to the head; and sample size [S] where (-) indicates a $5 \mathrm{~mm}$ block gauge and (+) a $10 \mathrm{~mm}$ block gauge used as samples.

\subsection{Experiment Plan and Results}

Table 2 shows a summary of results. F testing was carried out on the full results to determine the significance of factor interaction. F tests have been used for many years as a purely numerical method of deciding which effects are to be treated as real. In this case the $\mathrm{F}$ test ratios for the factor $\mathrm{L}$ and the LxD factor interaction were calculated and compared with the critical values for the particular degree of freedom. It was found that the factor L (Light source) should be treated as real. The LxD (Light source and sample Distance) interaction should be also treated as real. The most likely cause of the LxD deviating from the straight line of the expected distribution is the shadow projection angle created by the component when it is placed very close to the spot light. By excluding all the measurements that were taken by the spot light source, 
the general accuracy of the measurement was improved by $91 \%$ from $\pm 0.19 \mathrm{~mm}$ of the unmodified scanner system to accuracy of $\pm 0.1 \mathrm{~mm}$. Additionally, where the parallel beam of light was employed with no rotation of the component, the standard deviation of the runs $3,4,7,8,11$ and 12 was $\pm 0.052 \mathrm{~mm}$.

Table 2. Experiment plan and results showing setup of each run

\begin{tabular}{|c|c|c|c|c|c|c|c|}
\hline 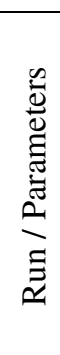 & 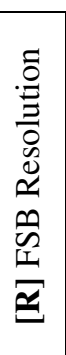 & 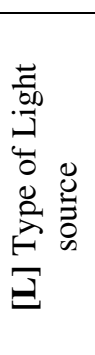 & 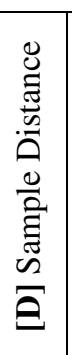 & 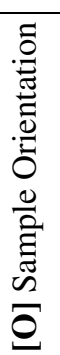 & 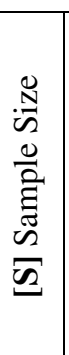 & 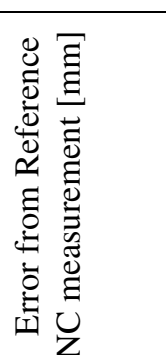 & 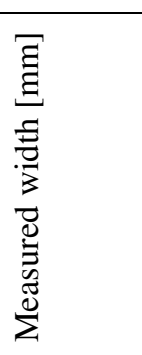 \\
\hline 1 & - & - & - & - & + & 0.311 & 10.287 \\
\hline 2 & + & - & - & - & - & 0.301 & 5.270 \\
\hline 3 & - & + & - & - & - & 0.068 & 5.037 \\
\hline 4 & + & + & - & - & + & 0.100 & 10.075 \\
\hline 5 & - & - & + & - & - & 0.787 & 5.757 \\
\hline 6 & + & - & + & - & + & 1.243 & 11.218 \\
\hline 7 & - & + & + & - & + & 0.015 & 9.990 \\
\hline 8 & + & + & + & - & - & -0.017 & 4.953 \\
\hline 9 & - & - & - & + & - & 0.322 & 5.291 \\
\hline 10 & + & - & - & + & + & 0.650 & 10.625 \\
\hline 11 & - & + & - & + & + & -0.027 & 9.948 \\
\hline 12 & + & + & - & + & - & -0.017 & 4.953 \\
\hline 13 & - & - & + & + & + & 1.581 & 11.557 \\
\hline 14 & + & - & + & + & - & 0.597 & 5.566 \\
\hline 15 & - & + & + & + & - & -0.229 & 4.741 \\
\hline 16 & + & + & + & + & + & -0.154 & 9.821 \\
\hline
\end{tabular}

\section{$4 \quad$ Results and Discussion}

\subsection{Initial Experiment}

The initial experiment consisted of eight runs and considered four factors at two levels. This was done to determine which of the four factors is significant and has an impact on the measurement accuracy. None of the four factors on their own noticeably influenced accuracy of the reading. However, interaction of factors D (distance of the sample) and S (size of the sample) has shown to be the main source of inaccuracy. The smaller sample (1 $\mathrm{mm}$ slot) placed $10 \mathrm{~mm}$ from the scanner surface resulted in the 
worst measurements with a standard deviation of $\pm 0.45 \mathrm{~mm}$. Calculating the standard deviation without the $\mathrm{D}$ and $\mathrm{S}$ factor interaction resulted in much better accuracy of $\pm 0.19 \mathrm{~mm}$ although this is still low compared to other metrology instruments.

It was observed from the pixel intensity curves that these poor accuracy levels were likely caused by two key factors. The first is that of the shadow projected behind the component by the scanner lamp during the scanning process. The shadow behind even a very thin $(1 \mathrm{~mm})$ sample creates dark areas which make it very difficult for the software to accurately identify the real position of the component edges. If the light intensity of the area is below $25 \%$ it is assumed to be part of the sample and adds several tenths of a millimetre to the measured dimension. On the other hand the width of a slot is reduced by this shadow. The effect of the shadow was slightly minimized by lifting the white backing board to some distance from the component so that the shadow would not be projected immediately behind the component. This modification brought unwanted reflections of the surrounding light from the metallic component that produced high intensity pixels (white) at the edges of the component. The second major cause of inaccuracy in the initial experiment was due to the component edges being beyond the scanner focus field, and therefore they were blurred. This is particularly evident for the scans with component edges further than $10 \mathrm{~mm}$ from the platen. The range where the real sample edge could be is wide and the $25 \%$ pixel intensity threshold does not identify the edge accurately. Such an error cannot be improved by increasing the scanning resolution.

\subsection{Second Stage Experiment}

The second stage experiment was designed to reduce the factors having a negative effect on the measurement accuracy identified in the initial experiment. Namely shadow, light reflection and scanner depth of the focus field. With a view to stretch the scanner measurement limits $5 \mathrm{~mm}$ thick slip gauges were used as samples for the second stage experiment instead of the thin sheet of metal. The factors used during initial experiment were kept also for the second stage experiment since the resolution was significantly reduced from 4800dpi and 1200dpi to 1200dpi and 600dpi which could potentially introduce more variation in the readings. The distance factor was kept due to its possible effects on light dispersion at the edge of the component and creating a wider shadow. The orientation factor was also maintained.

Despite a lower resolution the new FBS system resulted in significantly improved accuracy compared to the initial system. The type of light source proved to be very significant. The parallel beam generated by the scanner lamp placed above the component is preferred to the spot light source generated by the LED. The accuracy of $\pm 0.05 \mathrm{~mm}$ was found for runs with the parallel beam of light and no tilting of the sample.

The beam from the scanner lamp was parallel only in one direction because the light spreads in a radial direction normal to a cylinder's curved surface. The light from a spot source spreads radially outwards normal to the surface of a sphere. Only lengths in a parallel orientation to the lamp would produce the most accurate results. Uneven distribution of the shadow across the scanned image can be observed in runs 15 and 16 of the second stage experiment due to a $30^{\circ}$ rotation of the component against the lamp axis. This effect could be diminished by using a completely parallel light source achieved, for example, by a collimating lens or by reflecting light from a parabolic mirror with a light source at its focal point. 


\section{Conclusions}

With an unmodified scanner the interaction of sample distance and sample size were identified as significant. The best achieved accuracy of the unmodified scanner was $\pm 0.19 \mathrm{~mm}$ for $13.5 \mathrm{~mm}$ and $1 \mathrm{~mm}$ wide apertures in a $0.1 \mathrm{~mm}$ thin metal sheet sample using scanner resolutions of 1200dpi and 4800dpi. A system was then modified to achieve higher accuracy. By removing the scanner's internal lamp and projecting a shadow of the component onto a diffuser layer, the light reflection at the component edge and the shadow behind the component effects were eliminated. A parallel light source was identified to be most effective providing the highest reading accuracy of $\pm 0.05 \mathrm{~mm}$ when the sample was parallel to the lamp axis. Resolution of the second experiment was $1200 \mathrm{dpi}$ and $600 \mathrm{dpi}$. This accuracy of $\pm 0.05 \mathrm{~mm}$ could not be further improved due to difficulty in precisely determining the position of the component edge. Edge shadows were blurred due to light dispersal at the component edges. Compared to the accuracy of conventional metrology instruments the flatbed scanner would be suitable for medium accuracy measurements in a workshop. However, it is suggested that by using a higher resolution scanner and by making further modifications its accuracy could be improved to $\pm 0.01 \mathrm{~mm}$ which would be more appropriate for manufacturing metrology.

\section{References}

1. Quinn, T.J.: Metrology, its role in today's world, BIPM Rapport - 94/5. The International Bureau of Weights and Measures (1994)

2. Cheng, W.Y., Cheng, P.C., et al.: The use of modified flatbed and film scanners for large specimen imaging. Scanning 23(2), 135 (2001)

3. Krout, K.E., et al.: High-resolution scanner for neuroanatomical analysis. Journal of Neuroscience Methods 113(1), 37-40 (2002)

4. Bond-Lamberty, B., Gower, S.T.: Estimation of stand-level leaf area for boreal bryophytes. Oecologia 151, 584-592 (2006)

5. Bond-Lamberty, B., Wang, C., Gower, S.T.: The use of multiple measurement techniques to refine estimates of conifer needle geometry. Can. J. For. Res. 33, 101-105 (2003)

6. Kuchenbuch, R.O., Ingram, K.T.: Image analysis for non-destructive and non-invasive quantification of root growth and soil water content in rhizotrons. Journal of Plant Nutrition and Soil Science 165(5), 573-581 (2002)

7. Gabrielson, J., Hart, M., et al.: Evaluation of redox indicators and the use of digital scanners and spectrophotometer for quantification of microbial growth in microplates. Journal of Microbiological Methods 50(1), 63-73 (2002)

8. Wolf, R.E., Williams, W.L., et al.: Using 'DropletScan' to analyze spray quality. MidCentral ASAE (paper no. MC00-105), St. Joseph, MO (2000)

9. Peterson, K.W., Swartz, R.A., et al.: Hardened concrete air void analysis with a flatbed scanner. Transportation Research Record 1775, 36-43 (2001)

10. Miriello, D., Crisci, G.M.: Image analysis and flatbed scanners: A visual procedure in order to study the macroporosity of the archeological and historical mortals. Journal of Cultural Heritage 7, 186-192 (2006)

11. Van Dalen, G.: Determination of the size distribution and percentage of broken kernels of rice using flatbed scanning and image analysis. Food Res. Int. 37, 51-58 (2004) 
12. Shanin, M.A., Symons, S.J.: A machine vision system for grading lentils. Canadian Biosystems Engineering 43, 7.7-7.14 (2001)

13. Stanley, D.W., Baker, K.W.: A simple laboratory exercise in food structure/texture relationships using flatbed scanner. Journal of Food Science Education 1, 6-9 (2002)

14. Zalocha, D., Kasperkiewicz, J.: Estimation of the structure of air entrained concrete using a flatbed scanner. Cement and Concrete research 35, 2041-2046 (2005)

15. Casado-Rojo, S., Lorenzana, H.E.: A modified commercial scanner as an image plate for table-top optical applications. Review of Scientific Instruments 80(1), 13104-13104 (2009)

16. Jones, M.P., Callahan, R.N., Bruce, R.D.: Flow Length Measurement of Injection Molded Spirals using a Flatbed Scanner. J. Industrial technology 017(1) (2011)

17. Kee, C.W., Ratnam, M.M.: A simple approach to fine wire diameter measurement using a high-resolution flatbed scanner. International Journal of Advanced Manufacturing Technology 40(9-10), 940-947 (2009)

18. Hunter, J.S., Hunter, W.G.: Statistics for Experiments, 2nd edn. G.E.P. Box, Wiley \& Sons, Inc. ( (2005)

19. ImageJ, http://rsb.info.nih.gov/ij/ (accessed August 30, 2011)

20. Kresic-Juric, S., Madej, D., Fadil, S.: Applications of Hidden Markov Models in Bar Code Decoding. Intl. J. Pattern Recognition Letters 27, 1665-1672 (2006)

21. Suchendra, M., Yiqing, B.Z., Walter, D.P.: A Genetic Algorithm-based Edge Detection Technique. In: IEEE Proceedings of the IJCNN 1993, October 25-29, vol. 3, pp. 2995-2999 (1993)

22. Chung-Chia, K., Wen-June, W.: A novel edge detection method based on the maximizing objective function. Pattern Recognition 40, 609-6018 (2007)

23. Goodman, J.W.: Introduction to Fourier Optics. McGraw-Hill, New York (1967)

24. Russ, J.C.: The Image processing handbook, 3rd edn. CRC Press, FL (1999)

25. Epson Perfection V500 scanner handbook, http: / / www. epson. co.uk

26. Born, M., Wolf, E.: Principles of Optics, 6th edn. Pergamon Press Ltd., Oxford (1986)

27. Shehrzad, Q.: Embeded image processing on the TMS320C6000 ${ }^{\mathrm{TM}}$ DSP Examples in Code Composer Studio $^{\mathrm{TM}}$ and MATLAB. Springer Science+Business Media, Inc. (2005)

28. Super Flux LED White, fact sheet (2010), http: / / www. rapidonline. com (January 2010)

29. Chugui, Y.V.: Optical dimensional metrology for 3D objects of constant thickness. Measurement 30, 19-31 (2000)

30. Chugui, Y.V.: Fraunhofer diffraction by volumetric bodies of constant thickness. J. Opt. Soc. Am. (1989) 